\title{
9-(2-羟基乙基)-6-烷氨基-8-甲氧基-2-烷硫基嘌呤的合成及其 抗血小板凝集活性
}

\author{
汪 蹈 杜洪光* \\ (北京化工大学理学院 北京 100029)
}

\begin{abstract}
摘要 以 6-氯鸟嘌呤(1)为原料, 与乙酸-2-澳-乙酯反应得到9-乙酰氧基乙基-2-氨基-6-氯嘌呤 (2), 2 经溴代得到 9-乙酰氧 基乙基-2-氨基-6-氯-8-溴嘌呤(3), 3 在甲醇中与甲醇钠反应得到 9-(2-羊基乙基)-2-氨基-8-甲氧基-6-氯嘌呤(4), 对 4 的羟 基进行乙酰化保护得到 9-乙酰氧基乙基-2-氨基-8-甲氧基-6-氯嘌呤(5), 5 经重氮化反应后再与二烷基二硫醚反应得到 9乙酰氧基乙基-8-甲氧基-2-烷硫基-6-氯嘌呤(6), 6 与胺进行亲核取代反应得到 9-乙酰氧基乙基-6-烷氨基-8-甲氧基-2-烷 硫基嘌呤(7), 7 的羟基脱保护后得到 9-(2-差基乙基)-6-烷氨基-8-甲氧基-2-烷硫基嘌呤(8). 合成了 24 个未见报道的化合 物 3 8, 它们的结构经 ${ }^{1} \mathrm{H} N \mathrm{NR},{ }^{13} \mathrm{C} N \mathrm{NMR}$, IR 及 HRMS 等手段得到表征, 并对 9 个目标化合物 8 进行了抗血小板凝集 活性测试.
\end{abstract}

关键词＼cjkstart嘌呤衍生物; 合成; 结构表征; 抗血小板凝集

\section{Synthesis of 6-Alkylamino-2-alkylthio-9-(2-hydroxyethyl)-8-methoxy- purines and Their Anti-platelet Aggregation Activities}

\author{
Wang, Tao Du, Hongguang* \\ (College of Science, Beijing University of Chemical Technology, Beijing 100029)
}

\begin{abstract}
Amino-6-chloropurine (1) was reacted with 2-bromoethyl acetate to yield 9-acetoxyethyl-2-amino-6-chloropurine (2), which was treated with NBS to afford 9-acetoxyethyl-2-amino-8-bromo-6-chloropurine (3). 3 was reacted with sodium methanol to afford 2-amino-6-chloro-9-(2-hydroxyethyl)-8-methoxypurine (4), and then $\mathbf{4}$ was treated with acetic anhydride to yield 9-acetoxyethyl-2-amino-6-chloro-8-methoxypurine (5). After that 5 was diazotized and reacted with dialkyl disulfide to yield 9-acetoxyethyl-2-alkylthio-6-chloro-8-methoxypurine (6) and $\mathbf{6}$ was treated with amine to afford 9-acetoxyethyl-6-alkylamino-2-alkylthio-8-methoxypurine (7). Deprotection of hydroxyl group in compound 7 provided target compound 8 . All 24 novel compounds $3 \sim 8$ were acquired and their structures were identified by $1 \mathrm{H}$ NMR, 13C NMR, IR and HRMS techniques. Besides, the anti-platelet aggregation activities of the nine target compounds were tested.
\end{abstract}

Keywords purine derivatives; synthesis; characterization; anti-platelet aggregation

血小板聚集是动脉硬化和血栓形成的重要原因 ${ }^{[1]}$, 当血小板被激活时，其中包含的二磷酸腺苷(ADP)立即 释放, 作用于血小板的 ADP 受体, 加快血小板的聚集 ${ }^{[2]}$. 结构上属于 ADP 类似物的嘌呤衍生物, 会与 ADP 竞争 受体, 产生竞争性抑制作用, 从而达到抗血小板凝集的 作用, 这是嘌呤衍生物具有抗血小板凝聚药物潜力的理 论依据. 目前, 已有许多这类化合物被发现具有抗血小 板凝集活性, 其中 AR-C69931MX(Cangrelor) ${ }^{[3,4]}$, AZD6140 (Ticagrelor) ${ }^{[5,6]}$, AR-C67085 $5^{[7,8]}$ 和 AR-C78511 $1^{[9,10]}$ 等
(如 Scheme 1 所示)已处于临床研究阶段或进入市场.

早在 1973 年, Kikugawa 等 ${ }^{[11]}$ 就报道了在嘌呤环 6位引入烷氨基以及在 2-位引入硫原子 ${ }^{[12]}$ 可以使其抗血 小板凝集活性提高. 在之后的几十年间，涌现出许多关 于由对嘌呤环的 2-位、6-位、9-位三个位置进行结构改 造所得到的嘌呤衍生物的合成及其抗血小板凝集活性 的报道，这其中也包括本课题组吴兆军 ${ }^{[13]}$ 和何琦文 等 ${ }^{[14]}$ 的工作. 但有关在嘌呤环 8 -位有取代基的嘌呤衍生 物的报道较为少见，在该位置上引入取代基对化合物抗

*E-mail: dhg@mail.buct.edu.cn

Received March 12, 2013; revised April 11, 2013; published online April 24, 2013.

Project supported by the National Natural Science Foundation of China (No. 21272022).

国家自然科学基金(No. 21272022)资助项目. 
<smiles>CSCCNc1nc(SCCC(F)(F)F)nc2c1ncn2C1OCC(OP(=O)([O-])OP(=O)([O-])C(Cl)(Cl)P(=O)([O-])[O-])C(O)C1O</smiles>

AR-C69931MX(Cangrelor)<smiles>CCCSc1nc(N)c2ncn(C3OCC(O)C(OP(=O)(O)OP(=O)(O)C(Cl)(Cl)P(=O)(O)O)C3O)c2n1</smiles>

AR-C67085<smiles>CCCSc1nc(N[C@@H]2C[C@H]2c2ccc(F)c(F)c2)c2nnn(C3CCC(O)C(COCCO)C3O)c2n1</smiles>

AZD6140(Ticagrelor)<smiles>CCCCCCNc1nc(SCCC)nc2c1nnn2C1OC2C(=CC(=O)N[C@@H](CC(=O)O)C(=O)O)OC1C(O)C2O</smiles>

AR-C78511

Scheme 1

血小板凝聚活性的影响也尚不明确. 因此, 本文设计了 一类新型的在 2-位、6-位、8-位和 9-位四个位置上有取 代基的嘌呤衍生物(化合物 8), 对其合成及抗血小板凝 集活性进行研究. 合成路线如 Scheme 2 所示.

\section{1 结果与讨论}

\section{1 化合物 4 的合成}

对化合物 4 的合成进行研究发现, 反应过程中首先 生成了中间体 $4 \mathrm{a}$, 随着反应的进行, 出现了中间体 $\mathbf{4 b}$ 和 $4 \mathbf{c}$, 之后中间体 $4 \mathrm{a}$ 消失, 最后中间体 $\mathbf{4 c}$ 也逐渐消失, 只剩中间体 $\mathbf{4 b}$. 结构鉴定表明中间体 $4 \mathrm{a}$ 为化合物 3 中
乙酰基保护脱除产物 $\left[{ }^{1} \mathrm{H} \mathrm{NMR}\left(\mathrm{CDCl}_{3}, 400 \mathrm{MHz}\right) \delta: 5.35\right.$ (t, $J=4.77 \mathrm{~Hz}, 1 \mathrm{H}, \mathrm{OH}) ; 5.13\left(\mathrm{~s}, 2 \mathrm{H}, \mathrm{NH}_{2}\right) ; 4.31(\mathrm{t}, J=$ $5.08 \mathrm{~Hz}, 2 \mathrm{H}, \mathrm{CH}_{2} \mathrm{O}$ ); 3.72 (t, $\left.J=5.65 \mathrm{~Hz}, 2 \mathrm{H}, \mathrm{NCH}_{2}\right) ;{ }^{13} \mathrm{C}$ NMR $\left(\mathrm{CDCl}_{3}, 100 \mathrm{MHz}\right) \delta: 159.03,155.36,149.52$, 129.13, 124.61, 57.51, 47.92; HRMS (ESI) calcd for $\mathrm{C}_{7} \mathrm{H}_{8} \mathrm{BrClN}_{5} \mathrm{O}[\mathrm{M}+\mathrm{H}]^{+}$291.9601, found 291.9607], 中 间体 $4 \mathrm{~b}$ 为目标产物 4 , 中间体 $4 \mathrm{c}$ 为中间体 $4 \mathrm{a}$ 分子内亲 核取代的产物 $\left[{ }^{1} \mathrm{H}\right.$ NMR $\left(\mathrm{CDCl}_{3}, 400 \mathrm{MHz}\right) \delta: 5.18(\mathrm{t}, J=$ $\left.7.69 \mathrm{~Hz}, 2 \mathrm{H}, \mathrm{CH}_{2} \mathrm{O}\right) ; 4.92\left(\mathrm{~s}, 2 \mathrm{H}, \mathrm{NH}_{2}\right) ; 4.31$ (t, $J=7.76$ $\left.\mathrm{Hz}, 2 \mathrm{H}, \mathrm{NCH}_{2}\right) ;{ }^{13} \mathrm{C} \mathrm{NMR}\left(\mathrm{CDCl}_{3}, 100 \mathrm{MHz}\right) \delta: 59.07$, 155.72, 154.02, 147.64, 124.21, 64.32, 50.84; HRMS (ESI)<smiles>Nc1nc(Cl)c2nc(CC(=O)OCc3ccccc3)[nH]c2n1</smiles><smiles>CC(=O)OCCn1cnc2c(Cl)nc(N)nc21</smiles>

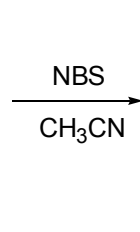<smiles>CC(=O)OCCn1c(Br)nc2c(Cl)nc(N)nc21</smiles>
$\underset{\mathrm{MeOH}}{\stackrel{\mathrm{MeONa}}{\longrightarrow}}$<smiles>CCOc1cc2c(Cl)nc(N)nc2n1CCO</smiles>

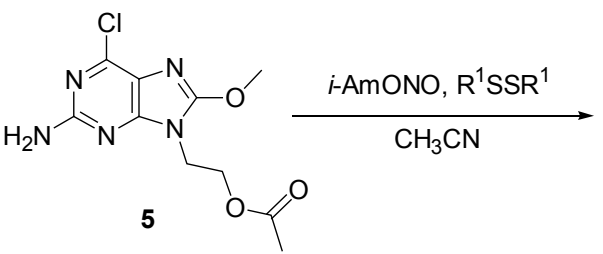

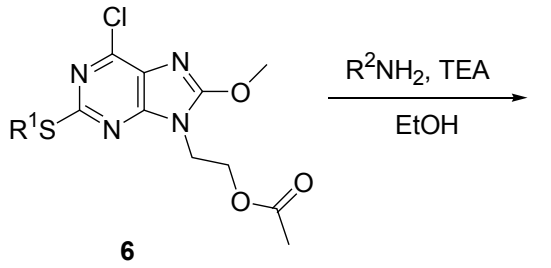

$\mathrm{R}^{1}=\mathrm{Et}, n-\mathrm{Pr}, n-\mathrm{Bu} ; \mathrm{R}^{2}=n-\mathrm{Pr}, n-\mathrm{Bu}, p-\mathrm{FC}_{6} \mathrm{H}_{4} \mathrm{CH}_{2} \mathrm{CH}_{2}$<smiles>[R]Nc1nc([R15])nc2c1nc(OC)n2CCOC(C)=O</smiles>

Scheme 2 
calcd for $\mathrm{C}_{7} \mathrm{H}_{7} \mathrm{ClN}_{5} \mathrm{O}[\mathrm{M}+\mathrm{H}]^{+}$212.0339, found 212.0337). 反应历程如 Scheme 3 所示.<smiles>CO[N+]([O-])(O)OC(C)=O</smiles><smiles>CCCn1c(OC)nc2c(Cl)nc(N)nc21</smiles>

\section{2 化合物 8 的波谱分析}

以化合物 $8 \mathbf{a}_{1}$ 为例, ${ }^{1} \mathrm{H}$ NMR 谱图中 $\delta 5.42$ 处的一质 子的单峰归属于 6-位烷氨基的 $\mathrm{NH}, \delta 5.04$ 处一质子的单 峰归属于 9-位碳链末端的 $\mathrm{OH}, \delta 4.09$ 处三质子的单峰归 属于 8-位 $\mathrm{OCH}_{3}, \delta 4.11 \sim 3.97$ 处四质子的两个三重峰归 属于 9-位碳链上的 $\mathrm{CH}_{2} \mathrm{CH}_{2}, \delta 3.58 \sim 3.57$ 处两质子的四 重峰归属于与 6-位正丙氨基氮原子相连的第一个 $\mathrm{CH}_{2}, \delta$ $3.14 \sim 3.08$ 处两质子的四重峰归属于 $\mathrm{CH}_{2}-\mathrm{S}, \delta 1.70 \sim$ 1.65 处两质子的六重峰归属于与 6-位正丙氨基氮原子 相连的第二个 $\mathrm{CH}_{2}, \delta 1.40$ 处三质子的三重峰归属于 2位乙硫基的 $\mathrm{CH}_{3}, \delta 1.00$ 处三质子的三重峰归属于 6-位 正丙氨基的 $\mathrm{CH}_{3} .{ }^{13} \mathrm{C}$ NMR 谱图中 $\delta 162.66,153.92$, 152.44，150.45，112.43 代表了嘌呤环碳骨架, $\delta 61.24$, 42.54 代表了 9-位直链碳骨架, $\delta 56.83$ 代表 8-位甲氧基 碳， $\delta 45.82,23.18,11.56$ 代表 6-位正丙氨基碳骨架， $\delta$ $32.17,14.52$ 代表 2-位乙硫基碳骨架. IR 谱图中, 3603 和 $3304 \mathrm{~cm}^{-1}$ 处的峰分别为 $\mathrm{OH}$ 和 $\mathrm{NH}$ 的伸缩振动, 2925 , $2863 \mathrm{~cm}^{-1}$ 以及 $1462,1417 \mathrm{~cm}^{-1}$ 处的峰表明甲基和亚甲 基的存在, $1615 \mathrm{~cm}^{-1}$ 为嘌呤环内碳碳双键的伸缩振动, 1319 和 $1039 \mathrm{~cm}^{-1}$ 处的峰进一步证实了 9-位直链末端的 羟基, $1146 \mathrm{~cm}^{-1}$ 表明 8 -位甲氧基的存在, 784 和 755 $\mathrm{cm}^{-1}$ 处的峰分别为乙基和丙基的面内摇摆振动. HRMS 测量值与计算值的吻合更加验证了该化合物的结构.

\section{3 化合物 8 的抗血小板凝集活性}

进行抗血小板凝集活性测试所用的方法为 WP (Washed Platelet)测试法. 本测试选用 DMSO 为阴性对
照物，AR-C69931MX 为阳性对照物，通过获得的血小 板凝集率数据来评估化合物对 ADP 诱导血小板凝集的 拮抗作用.

首先, 将抗凝剂(每 $100 \mathrm{~mL}$ 水中加入 $2.5 \mathrm{~g}$ 柠檬酸 钠, $1.5 \mathrm{~g}$ 柠檬酸, $2 \mathrm{~g}$ 葡萄糖)与新鲜血液按体积比 $1: 6$ 配制并在 $37{ }^{\circ} \mathrm{C}$ 预热，之后在 $1500 \mathrm{r} / \mathrm{min}$ 离心 $20 \mathrm{~min}$. 取 上层血浆于 $4000 \mathrm{r} / \mathrm{min}$ 再次离心 $8 \mathrm{~min}$, 体系由上至下 分为血浆、血小板、红细胞(微量)三层. 抽去血浆, 加入 等体积的苔氏液(含 $1 / 2000$ 的腺苷二磷酸酶，用于除 $\mathrm{ADP}$ ，避免血小板提前对 ADP 产生耐受性)，小心搅匀， 避免扰动红细胞层. 抽取上层体系, 于 $37{ }^{\circ} \mathrm{C}$ 下静置 10 $\min$, 之后抽取 $400 \mu \mathrm{L}$ 置于血小板凝集仪样品管中, 待 基线平稳后，加入 $5 \mu \mathrm{L}$ 纤维蛋白原溶液, $2 \mu \mathrm{L}$ 用 DMSO 配制的 $2 \mathrm{mmol} / \mathrm{L}$ 样品溶液, $3 \mathrm{~min}$ 后加入 $4 \mu \mathrm{L}$ ADP (1 $\mathrm{mmol} / \mathrm{L})$, 直至基线再次水平, 如此完成一个样品的测 试.将样品谱图与空白对照物 DMSO 的谱图对比, 即可 算出该样品在浓度为 $10 \mu \mathrm{mol} / \mathrm{L}$ 时的血小板凝集率.

9 个化合物的抗血小板凝集活性测试结果见表 1 . 测试结果显示，在嘌呤环 6-位取代基相同的情况下，2位取代基的活性顺序为正丙硫基 $>$ 正丁硫基 $>$ 乙硫基. 而在 2-位取代基相同的情况下，6-位取代基的活性顺序 为对氟苯乙氨基 $>$ 正丁氨基 $>$ 正丙氨基.

表 1 化合物 $8 \mathrm{a}_{1} \sim 8 \mathrm{c}_{3}$ 的抗血小板凝集活性数据 Table 1 Anti-platelet aggregation activities of compounds $8 \mathrm{a}_{1} \sim 8 \mathrm{c}_{3}$

\begin{tabular}{|c|c|c|c|}
\hline 样品名称 & $\mathrm{R}^{1}$ & $\mathrm{R}^{2}$ & 血小板凝集率 $/ \%$ \\
\hline $8 a_{1}$ & Et & $n-\operatorname{Pr}$ & 93.48 \\
\hline $8 \mathbf{a}_{2}$ & Et & $n$-Bu & 82.61 \\
\hline $8 \mathbf{a}_{3}$ & Et & $p-\mathrm{FC}_{6} \mathrm{H}_{4} \mathrm{CH}_{2} \mathrm{CH}_{2}$ & 76.22 \\
\hline $8 b_{1}$ & $n-\operatorname{Pr}$ & $n-\operatorname{Pr}$ & 71.74 \\
\hline $8 \mathbf{b}_{2}$ & $n-\operatorname{Pr}$ & $n-\mathrm{Bu}$ & 50.02 \\
\hline $8 b_{3}$ & $n-\operatorname{Pr}$ & $p-\mathrm{FC}_{6} \mathrm{H}_{4} \mathrm{CH}_{2} \mathrm{CH}_{2}$ & 43.48 \\
\hline $8 c_{1}$ & $n-\mathrm{Bu}$ & $n-\operatorname{Pr}$ & 78.26 \\
\hline $8 c_{2}$ & $n-\mathrm{Bu}$ & $n$-Bu & 65.22 \\
\hline $8 c_{3}$ & $n$-Bu & $p-\mathrm{FC}_{6} \mathrm{H}_{4} \mathrm{CH}_{2} \mathrm{CH}_{2}$ & 47.83 \\
\hline
\end{tabular}

\section{2 结论}

本文以 6-氯鸟嘌呤为原料, 合成出包括 9 个目标化 合物在内的 24 个未见报道的化合物. 抗血小板凝集活 性测试表明目标化合物具有一定的药理活性，但均与阳 性对照物 AR-C69931MX 相差较大(注：阳性对照物 AR-C69931MX 在 $10 \mu \mathrm{mol} / \mathrm{L}$ 时的血小板凝集率为 0 ).

\section{3 实验部分}

\section{1 仪器与试剂}

XT-4A 型显微熔点测定仪, 上海精密科学仪器有限 公司; 岛津-4000 型傅里叶变换红外光谱仪, 日本岛津 
公司; Bruker $400 \mathrm{MHz}$ 核磁共振仪; Agilent TOF-6210 型高分辨质谱仪, 美国安捷伦公司; TDZ5-WS 型低速离 心机, 湘仪离心机仪器有限公司; Model 400vs 型血小板 聚集仪, 美国 Chrono-Log 公司.

实验所用 6-氯鸟嘌呤为工业级, 其余原料如 2-溴乙 醇、NBS、甲醇钠、乙酸䣶、二烷基二硫醚、亚硝酸异 戊酯、伯胺以及各类溶剂等均为分析级. 乙酸-2-澳-乙酯 和 9-乙酰氧基乙基-6-氯-2-氨基嘌呤(2)按文献[14]方法 制备.

\section{2 实验方法}

3.2.19-乙酰氧基乙基-2-氨基-6-氯-8-溴嘌呤(3)的合 成

在装有冷凝管的三口圆底烧瓶中加入 $1 \mathrm{~g}(3.9$ $\mathrm{mmol}$ )化合物 $2,1.4 \mathrm{~g}(7.8 \mathrm{mmol}) \mathrm{NBS}$ 和 $60 \mathrm{~mL}$ 乙腈, 氮 气保护下加热回流 $2 \mathrm{~h}$. 用 $10 \%$ 硫代硫酸钠溶液洗涤, 再用乙酸乙酯萃取. 有机相用无水硫酸镁干燥, 过滤后 用柱层析色谱分离[展开剂为 $V$ (乙酸乙酯) : $V$ (石油 醚) $=1: 1$ ], 减压蒸出溶剂得到 $1.1 \mathrm{~g}$ 白色固体, 产率 $85 \%$. m.p. $104 \sim 106{ }^{\circ} \mathrm{C} ;{ }^{1} \mathrm{H}$ NMR $\left(\mathrm{CDCl}_{3}, 400 \mathrm{MHz}\right) \delta$ : $5.17\left(\mathrm{~s}, 2 \mathrm{H}, \mathrm{NH}_{2}\right), 4.43 \sim 4.37$ (m, 4H, $\left.\mathrm{NCH}_{2} \mathrm{CH}_{2} \mathrm{O}\right), 2.02$ $\left(\mathrm{s}, 3 \mathrm{H}, \mathrm{COCH}_{3}\right) ;{ }^{13} \mathrm{C} \mathrm{NMR}\left(\mathrm{CDCl}_{3}, 100 \mathrm{MHz}\right) \delta: 170.75$, $159.11,154.73,150.18,129.58,125.30,61.23,43.34$, 20.75; IR (KBr) v: 3334, 2923, 2863, 1707, 1616, 1449, 1357, 1236, $1054 \mathrm{~cm}^{-1}$; HRMS (ESI) calcd for $\mathrm{C}_{9} \mathrm{H}_{10} \mathrm{BrClN}_{5} \mathrm{O}_{2}[\mathrm{M}+\mathrm{H}]^{+}$333.9706, found 333.9700 .

3.2.29-(2-羟基乙基)-2-氨基-8-甲氧基-6-氯嘌呤(4) 的合成

在装有冷凝管的单口圆底烧瓶中加入 $1 \mathrm{~g}(3.0$ $\mathrm{mmol})$ 化合物 3, $0.35 \mathrm{~g}(6.6 \mathrm{mmol})$ 甲醇钠和 $50 \mathrm{~mL}$ 甲醇, 加热回流 $2 \mathrm{~h}$. 冷却至室温, 用稀盐酸调节 $\mathrm{pH}$ 至中性. 水洗, 用乙酸乙酯萃取, 有机相用无水硫酸美干燥, 过 滤后用柱层析色谱分离(展开剂为乙酸乙酯). 减压蒸出 溶剂得到 $660 \mathrm{mg}$ 白色固体, 产率 $90 \%$. m.p. $84 \sim 86{ }^{\circ} \mathrm{C}$; ${ }^{1} \mathrm{H} \mathrm{NMR}\left(\mathrm{CDCl}_{3}, 400 \mathrm{MHz}\right) \delta: 5.91$ (s, 2H, $\left.\mathrm{NH}_{2}\right), 3.70$ (s, $\left.3 \mathrm{H}, \mathrm{OCH}_{3}\right), 2.74(\mathrm{~s}, 1 \mathrm{H}, \mathrm{OH}), 2.69 \sim 2.52(\mathrm{~m}, 4 \mathrm{H}$, $\left.\mathrm{NCH}_{2} \mathrm{CH}_{2} \mathrm{O}\right) ;{ }^{13} \mathrm{C}$ NMR $\left(\mathrm{CDCl}_{3}, 100 \mathrm{MHz}\right) \delta: 159.01$, 156.46, 154.06, 147.43, 123.10, 60.54, 58.06, 43.53; IR (KBr) $v$ : 3603, 3347, 2935, 2867, 1632, 1465, 1381, 1341, $1138,1046 \mathrm{~cm}^{-1}$; HRMS (ESI) calcd for $\mathrm{C}_{8} \mathrm{H}_{11} \mathrm{ClN}_{5} \mathrm{O}_{2}$ $[\mathrm{M}+\mathrm{H}]^{+}$244.0601, found 244.0596.

3.2.39-乙酰氧基乙基-2-氨基-8-甲氧基-6-氯嘌呤(5) 的合成

在单口圆底烧瓶中加入 $1 \mathrm{~g}(4.1 \mathrm{mmol})$ 化合物 4, 0.5 $\mathrm{g}(4.9 \mathrm{mmol})$ 乙酸酎, $0.5 \mathrm{~g}(4.9 \mathrm{mmol})$ 三乙胺, 催化量的 DMAP 和 $60 \mathrm{~mL}$ 二氯甲烷, 室温搅拌 $1 \mathrm{~h}$. 水洗, 并用饱
和碳酸氢钠溶液调至中性, 用乙酸乙酯萃取, 有机相用 无水硫酸镁干燥, 过滤, 减压蒸出溶剂得到 $1.1 \mathrm{~g}$ 白色固 体, 产率 94\%. m.p. $115 \sim 117{ }^{\circ} \mathrm{C} ;{ }^{1} \mathrm{H}$ NMR $\left(\mathrm{CDCl}_{3}, 400\right.$ MHz) $\delta: 5.11\left(\mathrm{~s}, 2 \mathrm{H}, \mathrm{NH}_{2}\right), 4.36(\mathrm{t}, J=5.39 \mathrm{~Hz}, 2 \mathrm{H}$, $\mathrm{CH}_{2} \mathrm{O}$ ), 4.22 (s, 3H, $\mathrm{OCH}_{3}$ ), 4.18 (t, $J=5.39 \mathrm{~Hz}, 2 \mathrm{H}$, $\left.\mathrm{NCH}_{2} \mathrm{CH}_{2} \mathrm{O}\right), 1.99$ (s, 3H, $\left.\mathrm{COCH}_{3}\right) ;{ }^{13} \mathrm{C}$ NMR $\left(\mathrm{CDCl}_{3}, 100\right.$ MHz) $\delta: 170.71,157.94,156.83,154.40,146.31,121.97$, 61.24, 57.58, 40.28, 20.69; IR (KBr) v: 3308, 2941, 2866, 1712, 1628, 1451, 1356, 1234, 1139, $1048 \mathrm{~cm}^{-1}$; HRMS (ESI) calcd for $\mathrm{C}_{10} \mathrm{H}_{13} \mathrm{ClN}_{5} \mathrm{O}_{3}[\mathrm{M}+\mathrm{H}]^{+}$286.0707, found 286.0710 .

3.2.49-乙酰氧基乙基-8-甲氧基-2-烷硫基-6-氯嘌呤 (6)的合成

在装有冷凝管的三口圆底烧瓶中加入化合物 $1 \mathrm{~g}$ (3.5 mmol)化合物 $\mathbf{5}, 2.14 \mathrm{~g}(17.5 \mathrm{mmol})$ 二乙基二硫醚, $4.10 \mathrm{~g}(35 \mathrm{mmol})$ 亚硝酸异戊酯和 $50 \mathrm{~mL}$ 乙腈, 氮气保护 下 $70{ }^{\circ} \mathrm{C}$ 加热回流 $2 \mathrm{~h}$. 水洗, 用乙酸乙酯萃取, 有机相 用无水硫酸镁干燥, 过滤后用柱层析色谱分离[展开剂 为 $V$ (乙酸乙酯) $: V$ (石油醚 $)=2: 1$, 减压蒸出溶剂得到 化合物 6a. 参照上述方法用二正丙基二硫醚、二正丁基 二硫醚分别代替二乙基二硫醚可制得化合物 $\mathbf{6 b}$ 和 $\mathbf{6 c}$.

9-乙酰氧基乙基-8-甲氧基-2-乙硫基-6-氯嘌呤 $(6 \mathbf{a})$ : 白色固体, 产率 27\%. m.p. 84 85 ${ }^{\circ} \mathrm{C} ;{ }^{1} \mathrm{H}$ NMR $\left(\mathrm{CDCl}_{3}\right.$, $400 \mathrm{MHz}) \delta: 4.39\left(\mathrm{t}, J=5.27 \mathrm{~Hz}, 2 \mathrm{H}, \mathrm{CH}_{2} \mathrm{O}\right), 4.28(\mathrm{t}, J=$ $\left.5.27 \mathrm{~Hz}, 2 \mathrm{H}, \mathrm{NCH}_{2} \mathrm{CH}_{2} \mathrm{O}\right), 4.27\left(\mathrm{~s}, 3 \mathrm{H}, \mathrm{OCH}_{3}\right), 3.22 \sim$ 3.16 (q, $\left.J=7.33 \mathrm{~Hz}, 2 \mathrm{H}, \mathrm{SCH}_{2}\right), 1.98$ (s, $3 \mathrm{H}, \mathrm{COCH}_{3}$ ), $1.42\left(\mathrm{t}, J=7.37 \mathrm{~Hz}, 3 \mathrm{H}, \mathrm{SCH}_{2} \mathrm{CH}_{3}\right) ;{ }^{13} \mathrm{C} \mathrm{NMR}\left(\mathrm{CDCl}_{3}\right.$, $100 \mathrm{MHz}) \delta$ : 170.60, 163.32, 157.84, 153.30, 145.99, $125.87,61.17,57.94,40.62,25.83,20.65,14.37$; IR (KBr) $v: 2959,2873,1745,1613,1484,1411,1230,1142,1058$, $778 \mathrm{~cm}^{-1}$; HRMS (ESI) calcd for $\mathrm{C}_{12} \mathrm{H}_{16} \mathrm{ClN}_{4} \mathrm{O}_{3} \mathrm{~S}[\mathrm{M}+$ $\mathrm{H}]^{+}$331.0632, found 331.0638 .

9-乙酰氧基乙基-8-甲氧基-2-正丙硫基-6-氯嘌呤 (6b): 白色固体, 产率 $25 \%$. m.p. 86 87 ${ }^{\circ} \mathrm{C} ;{ }^{1} \mathrm{H}$ NMR $\left(\mathrm{CDCl}_{3}, 400 \mathrm{MHz}\right) \delta: 4.40\left(\mathrm{t}, J=5.30 \mathrm{~Hz}, 2 \mathrm{H}, \mathrm{CH}_{2} \mathrm{O}\right)$, $4.29\left(\mathrm{t}, J=5.30 \mathrm{~Hz}, 2 \mathrm{H}, \mathrm{NCH}_{2} \mathrm{CH}_{2} \mathrm{O}\right), 4.27\left(\mathrm{~s}, 3 \mathrm{H}, \mathrm{OCH}_{3}\right)$, 3.16 (t, $J=7.13 \mathrm{~Hz}, 2 \mathrm{H}, \mathrm{SCH}_{2}$ ), 1.98 (s, 3H, $\mathrm{COCH}_{3}$ ), $1.83 \sim 1.74\left(\mathrm{~m}, 2 \mathrm{H}, \mathrm{SCH}_{2} \mathrm{CH}_{2}\right), 1.06(\mathrm{t}, J=7.33 \mathrm{~Hz}, 3 \mathrm{H}$, $\left.\mathrm{SCH}_{2} \mathrm{CH}_{2} \mathrm{CH}_{3}\right) ;{ }^{13} \mathrm{C} \mathrm{NMR}\left(\mathrm{CDCl}_{3}, 100 \mathrm{MHz}\right) \delta: 170.55$, $163.41,157.81,153.24,145.84,125.79,61.14,57.92$, 40.62, 33.40, 22.52, 20.62, 13.46; IR (KBr) v: 2960, 2869, $1745,1612,1484,1347,1229,1141,1059,735 \mathrm{~cm}^{-1}$; HRMS (ESI) calcd for $\mathrm{C}_{13} \mathrm{H}_{18} \mathrm{ClN}_{4} \mathrm{O}_{3} \mathrm{~S} \quad[\mathrm{M}+\mathrm{H}]$ 345.0788, found 345.0790 .

9-乙酰氧基乙基-8-甲氧基-2-正丁硫基-6-氯嘌呤 
(6c): 白色固体, 产率 $22 \%$. m.p. 89 90 ${ }^{\circ} \mathrm{C} ;{ }^{1} \mathrm{H}$ NMR $\left(\mathrm{CDCl}_{3}, 400 \mathrm{MHz}\right) \delta: 4.39$ (t, $\left.J=5.16 \mathrm{~Hz}, 2 \mathrm{H}, \mathrm{CH}_{2} \mathrm{O}\right)$, 4.28 (t, $\left.J=5.16 \mathrm{~Hz}, 2 \mathrm{H}, \mathrm{NCH}_{2} \mathrm{CH}_{2} \mathrm{O}\right), 4.27\left(\mathrm{~s}, 3 \mathrm{H}, \mathrm{OCH}_{3}\right)$, $3.18\left(\mathrm{t}, J=7.29 \mathrm{~Hz}, 2 \mathrm{H}, \mathrm{SCH}_{2}\right), 1.98\left(\mathrm{~s}, 3 \mathrm{H}, \mathrm{COCH}_{3}\right)$, $1.78 \sim 1.70\left(\mathrm{~m}, 2 \mathrm{H}, \mathrm{SCH}_{2} \mathrm{CH}_{2}\right), 1.54 \sim 1.45(\mathrm{~m}, 2 \mathrm{H}$, $\mathrm{SCH}_{2} \mathrm{CH}_{2} \mathrm{CH}_{2}$ ), 0.96 (t, $J=7.29 \mathrm{~Hz}, 3 \mathrm{H}, \mathrm{SCH}_{2} \mathrm{CH}_{2} \mathrm{CH}_{2}-$ $\left.\mathrm{CH}_{3}\right) ;{ }^{13} \mathrm{C}$ NMR $\left(\mathrm{CDCl}_{3}, 100 \mathrm{MHz}\right) \delta: 170.59,163.51$, 157.82, 153.27, 145.95, 125.84, 61.17, 57.93, 40.62, 38.88, 31.24, 22.00, 20.65, 13.67; IR (KBr) v: 2956, 2869, 1745, 1612, 1486, 1347, 1229, 1142, 1058, $726 \mathrm{~cm}^{-1}$; HRMS (ESI) calcd for $\mathrm{C}_{14} \mathrm{H}_{20} \mathrm{ClN}_{4} \mathrm{O}_{3} \mathrm{~S}[\mathrm{M}+\mathrm{H}]^{+}$359.0945, found 359.0948 .

3.2.59-乙酰氧基乙基-6-烷氨基-8-甲氧基-2-烷硫基 嘌呤(7)的合成

在装有冷凝管的单口圆底烧瓶中加入 $1 \mathrm{~g}(3.0$ $\mathrm{mmol})$ 化合物 6a、0.21 g (3.6 mmol)正丙胺、 $0.37 \mathrm{~g} \mathrm{(3.6}$ $\mathrm{mmol}$ 三乙胺和 $40 \mathrm{~mL}$ 乙醇, 加热回流 $2 \mathrm{~h}$. 水洗, 用乙 酸乙酯萃取, 有机相用无水硫酸镁干燥, 过滤后用柱层 析色谱分离(展开剂为乙酸乙酯), 减压蒸出溶剂得到化 合物 7 $a_{1}$. 参照上述方法分别用正丁胺和对氟苯乙胺代 替正丙胺制得化合物 $7 \mathbf{a}_{2}$ 和 $7 a_{3}$. 同理, 用化合物 $\mathbf{6 b}$ 分 别和上述胺反应可制得化合物 $7 \mathbf{b}_{1} \sim 7 \mathbf{b}_{3}$. 用化合物 $\mathbf{6 c}$ 分别和上述胺反应可制得化合物 $7 \mathbf{c}_{1} \sim 7 \mathbf{c}_{3}$.

9-乙酰氧基乙基-6-正丙氨基-8-甲氧基-2-乙硫基嘌 呤 $\left(7 \mathbf{a}_{1}\right)$ : 白色固体, 产率 $85 \%$. m.p. $124 \sim 125{ }^{\circ} \mathrm{C} ;{ }^{1} \mathrm{H}$ NMR $\left(\mathrm{CDCl}_{3}, 400 \mathrm{MHz}\right) \delta: 5.31(\mathrm{~s}, 1 \mathrm{H}, \mathrm{NH}), 4.36$ (t, $J=$ $\left.5.34 \mathrm{~Hz}, 2 \mathrm{H}, \mathrm{CH}_{2} \mathrm{O}\right), 4.20(\mathrm{t}, J=5.26 \mathrm{~Hz}, 2 \mathrm{H}$, $\mathrm{NCH}_{2} \mathrm{CH}_{2} \mathrm{O}$ ), $4.12\left(\mathrm{~s}, 3 \mathrm{H}, \mathrm{OCH}_{3}\right), 3.60 \sim 3.55$ (q, $J=6.52$ $\mathrm{Hz}, 2 \mathrm{H}, \mathrm{CH}_{2} \mathrm{NH}$ ), $3.17 \sim 3.11$ (q, J=7.32 Hz, $2 \mathrm{H}, \mathrm{CH}_{2} \mathrm{~S}$ ), 1.98 (s, $\left.3 \mathrm{H}, \mathrm{COCH}_{3}\right), 1.71 \sim 1.61\left(\mathrm{~m}, 2 \mathrm{H}, \mathrm{NCH}_{2} \mathrm{CH}_{2}\right), 1.40$ (t, $J=7.29 \mathrm{~Hz}, 3 \mathrm{H}, \mathrm{SCH}_{2} \mathrm{CH}_{3}$ ), 1.00 (t, $J=7.37 \mathrm{~Hz}, 3 \mathrm{H}$, $\left.\mathrm{NCH}_{2} \mathrm{CH}_{2} \mathrm{CH}_{3}\right) ;{ }^{13} \mathrm{C} \mathrm{NMR}\left(\mathrm{CDCl}_{3}, 100 \mathrm{MHz}\right) \delta: 170.72$, $163.21,156.01,154.43,145.94,112.48,61.53,56.82$, 45.92, 40.01, 33.12, 23.16, 22.08, 13.83, 11.54; IR (KBr) v: 3369, 2928, 2866, 1742, 1615, 1457, 1411, 1235, 1142, $1057,782,745 \mathrm{~cm}^{-1}$; HRMS (ESI) calcd for $\mathrm{C}_{15} \mathrm{H}_{24} \mathrm{~N}_{5} \mathrm{O}_{3} \mathrm{~S}$ $[\mathrm{M}+\mathrm{H}]^{+} 354.1600$, found 354.1600 .

9-乙酰氧基乙基-6-正丁氨基-8-甲氧基-2-乙硫基嘌 呤 $\left(7 \mathbf{a}_{2}\right)$ : 白色固体, 产率 94\%. m.p. 127 128 ${ }^{\circ} \mathrm{C} ;{ }^{1} \mathrm{H}$ NMR $\left(\mathrm{CDCl}_{3}, 400 \mathrm{MHz}\right) \delta: 5.29$ (s, 1H, NH), 4.36 (t, $J=$ $5.45 \mathrm{~Hz}, 2 \mathrm{H}, \mathrm{CH}_{2} \mathrm{O}$ ), 4.19 (t, $J=5.45 \mathrm{~Hz}, 2 \mathrm{H}$, $\left.\mathrm{NCH}_{2} \mathrm{CH}_{2} \mathrm{O}\right), 4.11\left(\mathrm{~s}, 3 \mathrm{H}, \mathrm{OCH}_{3}\right), 3.63 \sim 3.58(\mathrm{q}, J=6.65$ $\mathrm{Hz}, 2 \mathrm{H}, \mathrm{CH}_{2} \mathrm{NH}$ ), $3.17 \sim 3.11$ (q, J=7.36 Hz, $2 \mathrm{H}, \mathrm{CH}_{2} \mathrm{~S}$ ), $1.98\left(\mathrm{~s}, 3 \mathrm{H}, \mathrm{COCH}_{3}\right), 1.67 \sim 1.60\left(\mathrm{~m}, 2 \mathrm{H}, \mathrm{NCH}_{2} \mathrm{CH}_{2}\right)$, $1.48 \sim 1.38\left(\mathrm{~m}, 5 \mathrm{H}, \mathrm{NCH}_{2} \mathrm{CH}_{2} \mathrm{CH}_{2}, \mathrm{SCH}_{2} \mathrm{CH}_{3}\right), 0.96(\mathrm{t}$,
$\left.J=7.36 \mathrm{~Hz}, 3 \mathrm{H}, \mathrm{NCH}_{2} \mathrm{CH}_{2} \mathrm{CH}_{2} \mathrm{CH}_{3}\right) ;{ }^{13} \mathrm{C}$ NMR $\left(\mathrm{CDCl}_{3}\right.$, $100 \mathrm{MHz}) \delta: 170.77,162.53,154.27,152.51,150.05$, $112.56,61.68,56.77,40.54,39.93,32.11,25.54,20.74$, 20.11, 14.99, 13.85; IR (KBr) v: 3377, 2929, 2866, 1743, $1615,1457,1412,1234,1140,1056,781,727 \mathrm{~cm}^{-1}$; HRMS (ESI) calcd for $\mathrm{C}_{16} \mathrm{H}_{26} \mathrm{~N}_{5} \mathrm{O}_{3} \mathrm{~S}[\mathrm{M}+\mathrm{H}]^{+} 368.1756$, found 368.1756 .

9-乙酰氧基乙基-6-对氟苯乙氨基-8-甲氧基-2-乙硫 基嘌呤 $\left(7 \mathrm{a}_{3}\right)$ : 白色固体, 产率 $86 \%$. m.p. $143 \sim 144{ }^{\circ} \mathrm{C}$; ${ }^{1} \mathrm{H}$ NMR $\left(\mathrm{CDCl}_{3}, 400 \mathrm{MHz}\right) \delta: 7.18 \sim 7.01(\mathrm{~m}, 4 \mathrm{H}, \mathrm{Ph})$, 5.34 (s, 1H, NH), 4.32 (t, J=5.44 Hz, 2H, $\left.\mathrm{CH}_{2} \mathrm{O}\right), 4.19$ (t, $\left.J=5.43 \mathrm{~Hz}, 2 \mathrm{H}, \mathrm{NCH}_{2} \mathrm{CH}_{2} \mathrm{O}\right), 4.08\left(\mathrm{~s}, 3 \mathrm{H}, \mathrm{OCH}_{3}\right), 3.89 \sim$ 3.83 (q, $J=6.77 \mathrm{~Hz}, 2 \mathrm{H}, \mathrm{CH}_{2} \mathrm{NH}$ ), $3.17 \sim 3.12$ (q, $J=7.35$ $\left.\mathrm{Hz}, 2 \mathrm{H}, \mathrm{CH}_{2} \mathrm{~S}\right), 2.96\left(\mathrm{t}, J=7.21 \mathrm{~Hz}, 2 \mathrm{H}, \mathrm{CH}_{2} \mathrm{Ph}\right), 1.94$ (s, $\left.3 \mathrm{H}, \mathrm{COCH}_{3}\right), 1.43\left(\mathrm{t}, J=7.22 \mathrm{~Hz}, 3 \mathrm{H}, \mathrm{SCH}_{2} \mathrm{CH}_{3}\right) ;{ }^{13} \mathrm{C}$ NMR $\left(\mathrm{CDCl}_{3}, 100 \mathrm{MHz}\right) \delta: 170.86,164.02,160.11$, $155.02,152.55,151.08,135.11,130.28,130.21,124.07$, 115.41, 115.21, 62.68, 56.80, 45.13, 43.14, 35.03, 31.05, 20.01, 13.80; IR (KBr) v: 3371, 2946, 2867, 1741, 1616, 1461, 1403, 1231, 1141, 1051, 806, $776 \mathrm{~cm}^{-1}$; HRMS (ESI) calcd for $\mathrm{C}_{20} \mathrm{H}_{25} \mathrm{FN}_{5} \mathrm{O}_{3} \mathrm{~S}[\mathrm{M}+\mathrm{H}]^{+} 434.1662$, found 434.1660 .

9-乙酰氧基乙基-6-正丙氨基-8-甲氧基-2-正丙硫基 嘌呤 $\left(7 \mathbf{b}_{1}\right)$ : 白色固体, 产率 94\%. m.p. $126 \sim 127{ }^{\circ} \mathrm{C} ;{ }^{1} \mathrm{H}$ $\operatorname{NMR}\left(\mathrm{CDCl}_{3}, 400 \mathrm{MHz}\right) \delta: 5.31(\mathrm{~s}, 1 \mathrm{H}, \mathrm{NH}), 4.35$ (t, $J=$ $\left.5.43 \mathrm{~Hz}, 2 \mathrm{H}, \mathrm{CH}_{2} \mathrm{O}\right), 4.19$ (t, $J=5.41 \mathrm{~Hz}, 2 \mathrm{H}$, $\mathrm{NCH}_{2} \mathrm{CH}_{2} \mathrm{O}$ ), 4.10 (s, 3H, OCH ${ }_{3}$ ), 3.59 3.55 (q, $J=6.46$ $\mathrm{Hz}, 2 \mathrm{H}, \mathrm{CH}_{2} \mathrm{NH}$ ), 3.09 (t, J=7.28 Hz, 2H, $\mathrm{CH}_{2} \mathrm{~S}$ ), 1.99 (s, $\left.3 \mathrm{H}, \mathrm{COCH}_{3}\right), 1.83 \sim 1.76\left(\mathrm{~m}, 2 \mathrm{H}, \mathrm{SCH}_{2} \mathrm{CH}_{2}\right), 1.74 \sim 1.66$ $\left(\mathrm{m}, 2 \mathrm{H}, \quad \mathrm{NCH}_{2} \mathrm{CH}_{2}\right), 1.05$ (t, $J=7.33 \mathrm{~Hz}, 3 \mathrm{H}$, $\mathrm{NCH}_{2} \mathrm{CH}_{2} \mathrm{CH}_{3}$ ), 1.00 (t, $J=7.54 \mathrm{~Hz}, 3 \mathrm{H}, \mathrm{SCH}_{2} \mathrm{CH}_{2} \mathrm{CH}_{3}$ ); ${ }^{13} \mathrm{C}_{\mathrm{NMR}}\left(\mathrm{CDCl}_{3}, 100 \mathrm{MHz}\right) \delta: 170.74,163.31,156.04$, 154.52, 146.02, 112.54, 61.54, 56.86, 45.94, 40.04, 33.14, 29.70, 23.21, 22.98, 13.57, 11.45; IR (KBr) v: 3382, 2926, 2861, 1742, 1618, 1461, 1392, 1241, 1151, 1049, 740 $\mathrm{cm}^{-1}$; HRMS (ESI) calcd for $\mathrm{C}_{16} \mathrm{H}_{26} \mathrm{~N}_{5} \mathrm{O}_{3} \mathrm{~S}[\mathrm{M}+\mathrm{H}]^{+}$ 368.1756 , found 368.1754 .

9-乙酰氧基乙基-6-正丁氨基-8-甲氧基-2-正丙硫基 嘌呤 $\left(7 \mathbf{b}_{2}\right)$ : 白色固体, 产率 $95 \%$. m.p. $132 \sim 133{ }^{\circ} \mathrm{C} ;{ }^{1} \mathrm{H}$ NMR $\left(\mathrm{CDCl}_{3}, 400 \mathrm{MHz}\right) \delta: 5.30$ (s, $\left.1 \mathrm{H}, \mathrm{NH}\right), 4.35$ (t, $J=$ $\left.5.41 \mathrm{~Hz}, 2 \mathrm{H}, \mathrm{CH}_{2} \mathrm{O}\right), 4.19$ (t, $J=5.41 \mathrm{~Hz}, 2 \mathrm{H}$, $\mathrm{NCH}_{2} \mathrm{CH}_{2} \mathrm{O}$ ), 4.11 (s, $3 \mathrm{H}, \mathrm{OCH}_{3}$ ), 3.63 3.58 (q, $J=6.67$ $\mathrm{Hz}, 2 \mathrm{H}, \mathrm{CH}_{2} \mathrm{NH}$ ), 3.11 (t, J=7.29 Hz, 2H, $\mathrm{CH}_{2} \mathrm{~S}$ ), 1.98 (s, $\left.3 \mathrm{H}, \mathrm{COCH}_{3}\right), 1.81 \sim 1.75\left(\mathrm{~m}, 2 \mathrm{H}, \mathrm{SCH}_{2} \mathrm{CH}_{2}\right), 1.68 \sim 1.60$ $\left(\mathrm{m}, 2 \mathrm{H}, \mathrm{NHCH}_{2} \mathrm{CH}_{2}\right), 1.46 \sim 1.41\left(\mathrm{~m}, 2 \mathrm{H}, \mathrm{NCH}_{2} \mathrm{CH}_{2} \mathrm{CH}_{2}\right.$ ), 
1.04 (t, $J=7.37 \mathrm{~Hz}, 3 \mathrm{H}, \mathrm{SCH}_{2} \mathrm{CH}_{2} \mathrm{CH}_{3}$ ), 0.96 (t, $J=7.37$ $\left.\mathrm{Hz}, 3 \mathrm{H}, \mathrm{NCH}_{2} \mathrm{CH}_{2} \mathrm{CH}_{2} \mathrm{CH}_{3}\right) ;{ }^{13} \mathrm{C} \mathrm{NMR}\left(\mathrm{CDCl}_{3}, 100 \mathrm{MHz}\right)$ $\delta: 170.61,162.24,154.05,152.43,150.02,112.34,62.85$, $56.73,40.52,39.91,36.75,31.61,24.13,20.71,20.02$, 14.04, 13.83; IR (KBr) v: 3394, 2923, 2859, 1730, 1618, 1457, 1381, 1266, 1178, 1048, 733, $726 \mathrm{~cm}^{-1}$; HRMS (ESI) calcd for $\mathrm{C}_{17} \mathrm{H}_{28} \mathrm{~N}_{5} \mathrm{O}_{3} \mathrm{~S}[\mathrm{M}+\mathrm{H}]^{+}$382.1913, found 382.1913.

9-乙酰氧基乙基-6-对氟苯乙氨基-8-甲氧基-2-正丙 硫基嘌呤 $\left(7 \mathbf{b}_{3}\right)$ : 白色固体, 产率 87\%. m.p. 147 148 ${ }^{\circ} \mathrm{C}$; ${ }^{1} \mathrm{H}$ NMR $\left(\mathrm{CDCl}_{3}, 400 \mathrm{MHz}\right) \delta: 7.20 \sim 6.93(\mathrm{~m}, 4 \mathrm{H}, \mathrm{Ph})$, $5.32(\mathrm{~s}, 1 \mathrm{H}, \mathrm{NH}), 4.36\left(\mathrm{t}, J=5.41 \mathrm{~Hz}, 2 \mathrm{H}, \mathrm{CH}_{2} \mathrm{O}\right), 4.20$ (t, $\left.J=5.34 \mathrm{~Hz}, 2 \mathrm{H}, \mathrm{NCH}_{2} \mathrm{CH}_{2} \mathrm{O}\right), 4.10\left(\mathrm{~s}, 3 \mathrm{H}, \mathrm{OCH}_{3}\right), 3.86 \sim$ 3.81 (q, $J=6.77 \mathrm{~Hz}, 2 \mathrm{H}, \mathrm{CH}_{2} \mathrm{NH}$ ), 3.13 (t, $J=7.22 \mathrm{~Hz}$, $2 \mathrm{H}, \mathrm{CH}_{2} \mathrm{~S}$ ), 2.94 (t, $\left.J=7.22 \mathrm{~Hz}, 2 \mathrm{H}, \mathrm{NCH}_{2} \mathrm{CH}_{2} \mathrm{Ph}\right), 1.99$ (s, $\left.3 \mathrm{H}, \mathrm{COCH}_{3}\right), 1.85 \sim 1.72\left(\mathrm{~m}, 2 \mathrm{H}, \mathrm{SCH}_{2} \mathrm{CH}_{2}\right), 1.04$ (t, $\left.J=7.37 \mathrm{~Hz}, 3 \mathrm{H}, \mathrm{SCH}_{2} \mathrm{CH}_{2} \mathrm{CH}_{3}\right) ;{ }^{13} \mathrm{C}$ NMR $\left(\mathrm{CDCl}_{3}, 100\right.$ MHz) $\delta: 170.76,163.94,160.13,154.82,152.52,151.04$, $135.10,130.20,124.14,115.41,115.21,64.06,56.80$, 43.47, 43.35, 39.52, 34.02, 22.76, 20.06, 13.73; IR (KBr) v: 3332, 2922, 2857, 1737, 1616, 1458, 1373, 1257, 1149, $1035,804,747 \mathrm{~cm}^{-1}$; HRMS (ESI) calcd for $\mathrm{C}_{21} \mathrm{H}_{27} \mathrm{~F}-$ $\mathrm{N}_{5} \mathrm{O}_{3} \mathrm{~S}[\mathrm{M}+\mathrm{H}]^{+}$448.1819, found 448.1817.

9-乙酰氧基乙基-6-正丙氨基-8-甲氧基-2-正丁硫基 嘌呤 $\left(\mathbf{7 c}_{1}\right)$ : 白色固体, 产率 94\%. m.p. 136 137 ${ }^{\circ} \mathrm{C} ;{ }^{1} \mathrm{H}$ NMR $\left(\mathrm{CDCl}_{3}, 400 \mathrm{MHz}\right) \delta: 5.29(\mathrm{~s}, 1 \mathrm{H}, \mathrm{NH}), 4.37$ (t, $J=$ $\left.5.46 \mathrm{~Hz}, 2 \mathrm{H}, \mathrm{CH}_{2} \mathrm{O}\right), 4.22$ (t, $J=5.45 \mathrm{~Hz}, 2 \mathrm{H}$, $\mathrm{NCH}_{2} \mathrm{CH}_{2} \mathrm{O}$ ), $4.10\left(\mathrm{~s}, 3 \mathrm{H}, \mathrm{OCH}_{3}\right), 3.58 \sim 3.56$ (q, $J=6.44$ $\mathrm{Hz}, 2 \mathrm{H}, \mathrm{NHCH}_{2}$ ), 3.10 (t, J=7.49 Hz, 2H, $\mathrm{SCH}_{2}$ ), 1.99 (s, $\left.3 \mathrm{H}, \mathrm{COCH}_{3}\right), 1.77 \sim 1.65\left(\mathrm{~m}, 4 \mathrm{H}, \mathrm{SCH}_{2} \mathrm{CH}_{2}, \mathrm{NCH}_{2}-\right.$ $\left.\mathrm{CH}_{2} \mathrm{CH}_{3}\right), 1.53 \sim 1.43\left(\mathrm{~m}, 2 \mathrm{H}, \mathrm{SCH}_{2} \mathrm{CH}_{2} \mathrm{CH}_{2}\right), 1.00$ (t, $J=$ $\left.7.35 \mathrm{~Hz}, 3 \mathrm{H}, \mathrm{NCH}_{2} \mathrm{CH}_{2} \mathrm{CH}_{3}\right), 0.95$ (t, $J=7.29 \mathrm{~Hz}, 3 \mathrm{H}$, $\left.\mathrm{SCH}_{2} \mathrm{CH}_{2} \mathrm{CH}_{2} \mathrm{CH}_{3}\right) ;{ }^{13} \mathrm{C}$ NMR $\left(\mathrm{CDCl}_{3}, 100 \mathrm{MHz}\right) \delta$ : $170.71,162.51,154.14,152.50,150.01,112.51,61.31$, $56.84,45.87,41.54,31.67,30.91,29.70,23.21,22.14$, 13.74, 11.44; IR (KBr) v: 3385, 2921, 2860, 1741, 1615, 1460, 1385, 1252, 1153, 1042, 742, $727 \mathrm{~cm}^{-1}$; HRMS (ESI) calcd for $\mathrm{C}_{17} \mathrm{H}_{28} \mathrm{~N}_{5} \mathrm{O}_{3} \mathrm{~S}[\mathrm{M}+\mathrm{H}]^{+}$382.1913, found 382.1916.

9-乙酰氧基乙基-6-正丁氨基-8-甲氧基-2-正丁硫基 嘌呤 $\left(\mathbf{7}_{2}\right)$ : 白色固体, 产率 $92 \%$. m.p. $138 \sim 139{ }^{\circ} \mathrm{C} ;{ }^{1} \mathrm{H}$ $\operatorname{NMR}\left(\mathrm{CDCl}_{3}, 400 \mathrm{MHz}\right) \delta: 5.26(\mathrm{~s}, 1 \mathrm{H}, \mathrm{NH}), 4.35$ (t, $J=$ $5.41 \mathrm{~Hz}, 2 \mathrm{H}, \mathrm{CH}_{2} \mathrm{O}$ ), 4.19 (t, $J=5.46 \mathrm{~Hz}, 2 \mathrm{H}, \mathrm{NCH}_{2} \mathrm{CH}_{2}-$ O), $4.11\left(\mathrm{~s}, 3 \mathrm{H}, \mathrm{OCH}_{3}\right), 3.63 \sim 3.58(\mathrm{q}, J=6.68 \mathrm{~Hz}, 2 \mathrm{H}$, $\mathrm{NHCH}_{2}$ ), 3.13 (t, $J=7.44 \mathrm{~Hz}, 2 \mathrm{H}, \mathrm{SCH}_{2}$ ), 1.98 (s, 3H,
$\left.\mathrm{COCH}_{3}\right), 1.78 \sim 1.59\left(\mathrm{~m}, 4 \mathrm{H}, \mathrm{SCH}_{2} \mathrm{CH}_{2}, \mathrm{NCH}_{2} \mathrm{CH}_{2} \mathrm{CH}_{2-}\right.$ $\left.\mathrm{CH}_{3}\right), \quad 1.52 \sim 1.48\left(\mathrm{~m}, 4 \mathrm{H}, \quad \mathrm{NCH}_{2} \mathrm{CH}_{2} \mathrm{CH}_{2} \mathrm{CH}_{3}, \mathrm{SCH}_{2}-\right.$ $\mathrm{CH}_{2} \mathrm{CH}_{2}$ ), 0.96 ( $\mathrm{t}, J=7.35 \mathrm{~Hz}, 3 \mathrm{H}, \mathrm{NCH}_{2} \mathrm{CH}_{2} \mathrm{CH}_{2}-\mathrm{CH}_{3}$ ), $0.88\left(\mathrm{t}, J=7.37 \mathrm{~Hz}, 3 \mathrm{H}, \mathrm{SCH}_{2} \mathrm{CH}_{2} \mathrm{CH}_{2} \mathrm{CH}_{3}\right) ;{ }^{13} \mathrm{C} \mathrm{NMR}$ $\left(\mathrm{CDCl}_{3}, 100 \mathrm{MHz}\right) \delta: 170.75,162.53,154.25,152.51$, $150.02,112.54,61.68,56.77,40.54,39.93,34.17,32.25$, $32.11,21.53,20.74,20.11,14.09,13.85$; IR (KBr) v: 3353, 2924, 2860, 1738, 1615, 1458, 1398, 1260, 1175, 1037, $729 \mathrm{~cm}^{-1}$; HRMS (ESI) calcd for $\mathrm{C}_{18} \mathrm{H}_{30} \mathrm{~N}_{5} \mathrm{O}_{3} \mathrm{~S}[\mathrm{M}+\mathrm{H}]^{+}$ 396.2069, found 396.2066.

9-乙酰氧基乙基-6-对氟苯乙氨基-8-甲氧基-2-正丁 硫基嘌呤 $\left(7 \mathbf{c}_{3}\right)$ : 白色固体, 产率 82\%. m.p. $153 \sim 154{ }^{\circ} \mathrm{C}$; ${ }^{1} \mathrm{H}$ NMR $\left(\mathrm{CDCl}_{3}, 400 \mathrm{MHz}\right) \delta: 7.22 \sim 6.97(\mathrm{~m}, 4 \mathrm{H}, \mathrm{Ph})$, $5.34(\mathrm{~s}, 1 \mathrm{H}, \mathrm{NH}), 4.36$ (t, $\left.J=5.41 \mathrm{~Hz}, 2 \mathrm{H}, \mathrm{CH}_{2} \mathrm{O}\right), 4.20(\mathrm{t}$, $\left.J=5.49 \mathrm{~Hz}, 2 \mathrm{H}, \mathrm{NCH}_{2} \mathrm{CH}_{2} \mathrm{O}\right), 4.10\left(\mathrm{~s}, 3 \mathrm{H}, \mathrm{OCH}_{3}\right), 3.87 \sim$ 3.81 (q, $\left.J=6.77 \mathrm{~Hz}, 2 \mathrm{H}, \mathrm{CH}_{2} \mathrm{NH}\right), 3.15$ (t, $J=7.44 \mathrm{~Hz}$, $2 \mathrm{H}, \mathrm{CH}_{2} \mathrm{~S}$ ), 2.94 (t, J=7.29 Hz, 2H, $\mathrm{CH}_{2} \mathrm{Ph}$ ), 1.99 (s, 3H, $\left.\mathrm{COCH}_{3}\right), 1.78 \sim 1.70\left(\mathrm{~m}, 2 \mathrm{H}, \mathrm{SCH}_{2} \mathrm{CH}_{2}\right), 1.52 \sim 1.45(\mathrm{~m}$, $2 \mathrm{H}, \mathrm{SCH}_{2} \mathrm{CH}_{2} \mathrm{CH}_{2}$ ), 0.94 (t, $J=7.44 \mathrm{~Hz}, 3 \mathrm{H}, \mathrm{SCH}_{2} \mathrm{CH}_{2-}$ $\left.\mathrm{CH}_{2} \mathrm{CH}_{3}\right) ;{ }^{13} \mathrm{C}$ NMR $\left(\mathrm{CDCl}_{3}, 100 \mathrm{MHz}\right) \delta: 170.73,163.22$, $160.13,154.52,152.14,150.51,135.11,130.27,130.18$, $124.10,115.41,115.19,64.36,56.79,43.52,42.46,39.94$, $35.48,31.84,22.15,20.03,13.73$; IR (KBr) v: 3385, 2924, 2863, 1729, 1616, 1457, 1384, 1263, 1178, 1047, 803, 727 $\mathrm{cm}^{-1}$; HRMS (ESI) calcd for $\mathrm{C}_{22} \mathrm{H}_{29} \mathrm{FN}_{5} \mathrm{O}_{3} \mathrm{~S}[\mathrm{M}+\mathrm{H}]^{+}$ 462.1975, found 462.1972 .

3.2.69-(2-差基乙基)-6-烷氨基-8-甲氧基-2-烷硫基 嘌呤 $(8)$ 的合成

在单口圆底烧瓶中加入 $1 \mathrm{~g}(2.8 \mathrm{mmol})$ 化合物 $7 \mathbf{a}_{1}$ 、 $0.17 \mathrm{~g}(3.1 \mathrm{mmol})$ 甲醇钠和 $40 \mathrm{~mL}$ 甲醇, 室温搅拌 $2 \mathrm{~h}$. 用稀盐酸调节 $\mathrm{pH}$ 至中性, 水洗, 用乙酸乙酯萃取, 有机 相用无水硫酸镁干燥, 减压蒸出溶剂得到化合物 $\mathbf{8} \mathbf{a}_{1}$. 参照上述方法分别用化合物 $7 \mathbf{a}_{2} \sim 7 \mathbf{c}_{3}$ 代替化合物 $7 \mathbf{a}_{1}$ 制 得化合物 $8 \mathrm{a}_{2} \sim 8 \mathrm{c}_{3}$.

9-(2-羟基乙基)-6-正丙氨基-8-甲氧基-2-乙硫基嘌 呤 $\left(\mathbf{8 a}_{1}\right)$ : 白色固体, 产率 $90 \%$. m.p. $115 \sim 116{ }^{\circ} \mathrm{C} ;{ }^{1} \mathrm{H}$ NMR $\left(\mathrm{CDCl}_{3}, 400 \mathrm{MHz}\right) \delta: 5.42(\mathrm{~s}, 1 \mathrm{H}, \mathrm{NH}), 5.04(\mathrm{~s}, 1 \mathrm{H}$, $\mathrm{OH}$ ), $4.11 \sim 4.08\left(\mathrm{~m}, 5 \mathrm{H}, \mathrm{CH}_{2} \mathrm{O}, \mathrm{OCH}_{3}\right.$ ), 3.97 (t, $J=5.11$ $\mathrm{Hz}, 2 \mathrm{H}, \mathrm{NCH}_{2} \mathrm{CH}_{2} \mathrm{O}$ ), $3.58 \sim 3.57$ (q, $J=6.41 \mathrm{~Hz}, 2 \mathrm{H}$, $\mathrm{CH}_{2} \mathrm{NH}$ ), $3.14 \sim 3.08$ (q, $J=7.27 \mathrm{~Hz}, 2 \mathrm{H}, \mathrm{CH}_{2} \mathrm{~S}$ ), $1.70 \sim$ $1.65\left(\mathrm{~m}, 2 \mathrm{H}, \mathrm{CH}_{2} \mathrm{CH}_{2} \mathrm{NH}\right), 1.40(\mathrm{t}, J=7.22 \mathrm{~Hz}, 3 \mathrm{H}$, $\left.\mathrm{SCH}_{2} \mathrm{CH}_{3}\right), 1.00$ (t, $\left.J=7.37 \mathrm{~Hz}, 3 \mathrm{H}, \mathrm{NHCH}_{2} \mathrm{CH}_{2} \mathrm{CH}_{3}\right) ;{ }^{13} \mathrm{C}$ NMR $\left(\mathrm{CDCl}_{3}, 100 \mathrm{MHz}\right) \delta$ : 162.66, 153.92, 152.44, $150.45,112.43,61.24,56.83,45.82,42.54,32.17,23.18$, 14.52, 11.56; IR (KBr) v: 3603, 3304, 2925, 2863, 1615, 
1462, 1417, 1319, 1146, 1039, 784, $755 \mathrm{~cm}^{-1}$; HRMS (ESI) calcd for $\mathrm{C}_{13} \mathrm{H}_{22} \mathrm{~N}_{5} \mathrm{O}_{2} \mathrm{~S}[\mathrm{M}+\mathrm{H}]^{+}$312.1494, found 312.1492 .

9-(2-羟基乙基)-6-正丁氨基-8-甲氧基-2-乙硫基嘌 呤 $\left(\mathbf{8 a}_{2}\right)$ : 白色固体, 产率 94\%. m.p. $118 \sim 119{ }^{\circ} \mathrm{C} ;{ }^{1} \mathrm{H}$ NMR $\left(\mathrm{CDCl}_{3}, 400 \mathrm{MHz}\right) \delta: 5.57$ (s, 1H, NH), 5.31 (s, 1H, $\mathrm{OH}), 4.08\left(\mathrm{t}, J=5.25 \mathrm{~Hz}, 2 \mathrm{H}, \mathrm{CH}_{2} \mathrm{O}\right), 4.01\left(\mathrm{~s}, 3 \mathrm{H}, \mathrm{OCH}_{3}\right)$, $3.98\left(\mathrm{t}, J=5.25 \mathrm{~Hz}, 2 \mathrm{H}, \mathrm{NCH}_{2} \mathrm{CH}_{2} \mathrm{O}\right.$ ), $3.61 \sim 3.56$ (q, $J=$ $6.54 \mathrm{~Hz}, 2 \mathrm{H}, \mathrm{CH}_{2} \mathrm{NH}$ ), 3.14 3.08 (q, J=7.29 Hz, $2 \mathrm{H}$, $\left.\mathrm{CH}_{2} \mathrm{~S}\right), 1.67 \sim 1.59\left(\mathrm{~m}, 2 \mathrm{H}, \mathrm{CH}_{2} \mathrm{CH}_{2} \mathrm{NH}\right), 1.46 \sim 1.37(\mathrm{~m}$, $\left.5 \mathrm{H}, \mathrm{CH}_{2} \mathrm{CH}_{2} \mathrm{CH}_{2} \mathrm{NH}, \mathrm{SCH}_{2} \mathrm{CH}_{3}\right), 0.96$ (t, $J=7.33 \mathrm{~Hz}, 3 \mathrm{H}$, $\left.\mathrm{NHCH}_{2} \mathrm{CH}_{2} \mathrm{CH}_{2} \mathrm{CH}_{3}\right) ;{ }^{13} \mathrm{C} \mathrm{NMR}\left(\mathrm{CDCl}_{3}, 100 \mathrm{MHz}\right) \delta$ : $162.47,153.99,152.49,149.62,112.42,61.13,56.81$, $45.72,40.50,29.70,25.44,20.08,14.72,13.84$; IR (KBr) $v: 3451,3293,2924,2861,1618,1464,1419,1317,1143$, 1062, 775, $728 \mathrm{~cm}^{-1}$; HRMS (ESI) calcd for $\mathrm{C}_{14} \mathrm{H}_{24} \mathrm{~N}_{5} \mathrm{O}_{2} \mathrm{~S}$ $[\mathrm{M}+\mathrm{H}]^{+}$326.1651, found 326.1650.

9-(2-差基乙基)-6-对氟苯乙氨基-8-甲氧基-2-乙硫 基嘌呤 $\left(\mathbf{8 a}_{3}\right)$ : 白色固体，产率 $92 \%$. m.p. 134 135 ${ }^{\circ} \mathrm{C}$; ${ }^{1} \mathrm{H}$ NMR $\left(\mathrm{CDCl}_{3}, 400 \mathrm{MHz}\right) \delta: 7.18 \sim 6.97(\mathrm{~m}, 4 \mathrm{H}, \mathrm{Ph})$, $5.42(\mathrm{~s}, 1 \mathrm{H}, \mathrm{NH}), 5.02(\mathrm{~s}, 1 \mathrm{H}, \mathrm{OH}), 4.08(\mathrm{t}, J=5.24 \mathrm{~Hz}$, $\left.2 \mathrm{H}, \mathrm{CH}_{2} \mathrm{O}\right), 4.09$ (s, 3H, $\left.\mathrm{OCH}_{3}\right), 3.96$ (t, $J=5.24 \mathrm{~Hz}, 2 \mathrm{H}$, $\left.\mathrm{NCH}_{2} \mathrm{CH}_{2} \mathrm{O}\right), 3.87 \sim 3.82\left(\mathrm{q}, J=6.61 \mathrm{~Hz}, 2 \mathrm{H}, \mathrm{CH}_{2} \mathrm{NH}\right)$, $3.15 \sim 3.10$ (q, $\left.J=7.29 \mathrm{~Hz}, 2 \mathrm{H}, \mathrm{CH}_{2} \mathrm{~S}\right), 2.94$ (t, $J=6.81$ $\left.\mathrm{Hz}, 2 \mathrm{H}, \mathrm{CH}_{2} \mathrm{Ph}\right), 1.41\left(\mathrm{t}, J=7.21 \mathrm{~Hz}, 3 \mathrm{H}, \mathrm{SCH}_{2} \mathrm{CH}_{3}\right) ;{ }^{13} \mathrm{C}$ NMR $\left(\mathrm{CDCl}_{3}, 100 \mathrm{MHz}\right) \delta: 164.06,160.13,155.07$, $152.58,151.26,135.10,130.28,130.20,124.14,115.42$, $115.21,61.65,56.81,46.52,43.51,35.54,32.01,13.82$; IR (KBr) v: 3482, 3307, 2926, 2858, 1616, 1451, 1405, 1332, $1148,1053,829,781 \mathrm{~cm}^{-1}$; HRMS (ESI) calcd for $\mathrm{C}_{18} \mathrm{H}_{23} \mathrm{FN}_{5} \mathrm{O}_{2} \mathrm{~S}[\mathrm{M}+\mathrm{H}]^{+}$392.1556, found 392.1554.

9-(2-羊基乙基)-6-正丙氨基-8-甲氧基-2-正丙硫基 嘌呤 $\left(\mathbf{8 b}_{1}\right)$ : 白色固体, 产率 $92 \%$. m.p. $119 \sim 120{ }^{\circ} \mathrm{C} ;{ }^{1} \mathrm{H}$ NMR $\left(\mathrm{CDCl}_{3}, 400 \mathrm{MHz}\right) \delta: 5.42(\mathrm{~s}, 1 \mathrm{H}, \mathrm{NH}), 5.06$ (s, 1H, $\mathrm{OH}), 4.08\left(\mathrm{t}, J=5.26 \mathrm{~Hz}, 2 \mathrm{H}, \mathrm{CH}_{2} \mathrm{O}\right), 4.06\left(\mathrm{~s}, 3 \mathrm{H}, \mathrm{OCH}_{3}\right)$, $3.97\left(\mathrm{t}, J=5.25 \mathrm{~Hz}, 2 \mathrm{H}, \mathrm{NCH}_{2} \mathrm{CH}_{2} \mathrm{O}\right.$ ), $3.59 \sim 3.55$ (q, $J=$ $6.37 \mathrm{~Hz}, 2 \mathrm{H}, \mathrm{CH}_{2} \mathrm{NH}$ ), 3.08 (t, $J=7.22 \mathrm{~Hz}, 2 \mathrm{H}, \mathrm{SCH}_{2}$ ), $1.82 \sim 1.73\left(\mathrm{~m}, 2 \mathrm{H}, \mathrm{SCH}_{2} \mathrm{CH}_{2}\right), 1.70 \sim 1.63(\mathrm{~m}, 2 \mathrm{H}$, $\mathrm{CH}_{2} \mathrm{CH}_{2} \mathrm{NH}$ ), 1.05 (t, $J=7.29 \mathrm{~Hz}, 3 \mathrm{H}, \mathrm{NHCH}_{2} \mathrm{CH}_{2} \mathrm{CH}_{3}$ ), $1.00\left(\mathrm{t}, J=7.52 \mathrm{~Hz}, 3 \mathrm{H}, \mathrm{SCH}_{2} \mathrm{CH}_{2} \mathrm{CH}_{3}\right) ;{ }^{13} \mathrm{C} \mathrm{NMR}$ $\left(\mathrm{CDCl}_{3}, 100 \mathrm{MHz}\right) \delta: 162.60,153.99,152.50,112.48$, $61.24,56.81,45.84,42.57,33.15,23.20,23.01,13.57$, 11.45; IR (KBr) v: 3609, 3317, 2924, 2863, 1616, 1462, 1414, 1319, 1146, 1037, $755 \mathrm{~cm}^{-1}$; HRMS (ESI) calcd for $\mathrm{C}_{14} \mathrm{H}_{24} \mathrm{~N}_{5} \mathrm{O}_{2} \mathrm{~S}[\mathrm{M}+\mathrm{H}]^{+}$326.1651, found 326.1651.
9-(2-羟基乙基)-6-正丁氨基-8-甲氧基-2-正丙硫基 嘌呤 $\left(\mathbf{8 b}_{2}\right)$ : 白色固体，产率 $90 \%$. m.p. 123 $124{ }^{\circ} \mathrm{C} ;{ }^{1} \mathrm{H}$ NMR $\left(\mathrm{CDCl}_{3}, 400 \mathrm{MHz}\right) \delta: 5.57(\mathrm{~s}, 1 \mathrm{H}, \mathrm{NH}), 5.48(\mathrm{~s}, 1 \mathrm{H}$, $\mathrm{OH}), 4.09 \sim 4.07\left(\mathrm{~m}, 5 \mathrm{H}, \mathrm{CH}_{2} \mathrm{O}, \mathrm{OCH}_{3}\right), 3.96(\mathrm{t}, J=5.24$ $\mathrm{Hz}, 2 \mathrm{H}, \mathrm{NCH}_{2} \mathrm{CH}_{2} \mathrm{O}$ ), $3.63 \sim 3.58$ (q, $J=6.47 \mathrm{~Hz}, 2 \mathrm{H}$, $\left.\mathrm{CH}_{2} \mathrm{NH}\right), 3.08\left(\mathrm{t}, J=7.22 \mathrm{~Hz}, 2 \mathrm{H}, \mathrm{CH}_{2} \mathrm{~S}\right), 1.82 \sim 1.73(\mathrm{~m}$, $\left.2 \mathrm{H}, \mathrm{SCH}_{2} \mathrm{CH}_{2}\right), 1.67 \sim 1.60\left(\mathrm{~m}, 2 \mathrm{H}, \mathrm{CH}_{2} \mathrm{CH}_{2} \mathrm{NH}\right), 1.50 \sim$ $1.40\left(\mathrm{~m}, 2 \mathrm{H}, \mathrm{CH}_{2} \mathrm{CH}_{2} \mathrm{CH}_{2} \mathrm{NH}\right), 1.04$ (t, $J=7.37 \mathrm{~Hz}, 3 \mathrm{H}$, $\left.\mathrm{SCH}_{2} \mathrm{CH}_{2} \mathrm{CH}_{3}\right), 0.96\left(\mathrm{t}, J=7.37 \mathrm{~Hz}, 3 \mathrm{H}, \mathrm{NHCH}_{2} \mathrm{CH}_{2}-\right.$ $\left.\mathrm{CH}_{2} \mathrm{CH}_{3}\right) ;{ }^{13} \mathrm{C} \mathrm{NMR}\left(\mathrm{CDCl}_{3}, 100 \mathrm{MHz}\right) \delta: 163.54,154.56$, 152.52, 150.06, 112.57, 61.68, 56.79, 43.56, 39.94, 33.24, 29.70, 23.23, 20.06, 13.86, 11.56; IR (KBr) v: 3472, 3312, 2925, 2861, 1617, 1461, 1415, 1321, 1144, 1045, 741, 726 $\mathrm{cm}^{-1}$; HRMS (ESI) calcd for $\mathrm{C}_{15} \mathrm{H}_{26} \mathrm{~N}_{5} \mathrm{O}_{2} \mathrm{~S}[\mathrm{M}+\mathrm{H}]^{+}$ 340.1807 , found 340.1810 .

9-(2-羟基乙基)-6-对氟苯乙氨基-8-甲氧基-2-正丙 硫基嘌呤 $\left(\mathbf{8 b}_{3}\right)$ : 白色固体，产率 93\%. m.p. 140 141 ${ }^{\circ} \mathrm{C}$; ${ }^{1} \mathrm{H}$ NMR $\left(\mathrm{CDCl}_{3}, 400 \mathrm{MHz}\right) \delta: 7.22 \sim 6.97(\mathrm{~m}, 4 \mathrm{H}, \mathrm{Ph})$, $5.32(\mathrm{~s}, 1 \mathrm{H}, \mathrm{NH}), 5.01(\mathrm{~s}, 1 \mathrm{H}, \mathrm{OH}), 4.34(\mathrm{t}, J=5.27 \mathrm{~Hz}$, $\left.2 \mathrm{H}, \mathrm{CH}_{2} \mathrm{O}\right), 4.32\left(\mathrm{~s}, 3 \mathrm{H}, \mathrm{OCH}_{3}\right), 4.21(\mathrm{t}, J=5.22 \mathrm{~Hz}, 2 \mathrm{H}$, $\mathrm{NCH}_{2} \mathrm{CH}_{2} \mathrm{O}$ ), $3.87 \sim 3.81$ (q, $J=6.62 \mathrm{~Hz}, 2 \mathrm{H}, \mathrm{CH}_{2} \mathrm{NH}$ ), $3.11\left(\mathrm{t}, J=7.13 \mathrm{~Hz}, 2 \mathrm{H}, \mathrm{CH}_{2} \mathrm{~S}\right), 2.92$ (t, $J=6.82 \mathrm{~Hz}, 2 \mathrm{H}$, $\left.\mathrm{CH}_{2} \mathrm{Ph}\right), 1.82 \sim 1.76\left(\mathrm{~m}, 2 \mathrm{H}, \mathrm{SCH}_{2} \mathrm{CH}_{2}\right), 1.04(\mathrm{t}, J=7.37$ $\left.\mathrm{Hz}, 3 \mathrm{H}, \mathrm{SCH}_{2} \mathrm{CH}_{2} \mathrm{CH}_{3}\right) ;{ }^{13} \mathrm{C} \mathrm{NMR}\left(\mathrm{CDCl}_{3}, 100 \mathrm{MHz}\right) \delta$ : $164.04,160.16,155.02,152.56,151.23,135.10,130.20$, $124.20,115.42,115.21,61.66,56.81,46.57,43.53,40.02$, $34.07,23.16,13.63$; IR (KBr) v: 3664, 3333, 2924, 2860, $1614,1455,1411,1319,1156,1069,825,747 \mathrm{~cm}^{-1}$; HRMS (ESI) calcd for $\mathrm{C}_{19} \mathrm{H}_{25} \mathrm{FN}_{5} \mathrm{O}_{2} \mathrm{~S}[\mathrm{M}+\mathrm{H}]^{+}$406.1713, found 406.1712 .

9-(2-差基乙基)-6-正丙氨基-8-甲氧基-2-正丁硫基 嘌呤 $\left(\mathbf{8 c}_{1}\right)$ : 白色固体, 产率 $91 \%$. m.p. $128 \sim 129{ }^{\circ} \mathrm{C} ;{ }^{1} \mathrm{H}$ NMR $\left(\mathrm{CDCl}_{3}, 400 \mathrm{MHz}\right) \delta: 5.40$ (s, 1H, NH), 4.97 (s, 1H, $\mathrm{OH}), 4.08\left(\mathrm{~m}, 5 \mathrm{H}, \mathrm{CH}_{2} \mathrm{O}, \mathrm{OCH}_{3}\right), 3.97$ (t, $J=5.24 \mathrm{~Hz}, 2 \mathrm{H}$, $\mathrm{NCH}_{2} \mathrm{CH}_{2} \mathrm{O}$ ), $3.58 \sim 3.56$ (q, $J=6.31 \mathrm{~Hz}, 2 \mathrm{H}, \mathrm{CH}_{2} \mathrm{NH}$ ), $3.10\left(\mathrm{t}, J=7.37 \mathrm{~Hz}, 2 \mathrm{H}, \mathrm{CH}_{2} \mathrm{~S}\right), 1.77 \sim 1.65\left(\mathrm{~m}, 4 \mathrm{H}, \mathrm{SCH}_{2}-\right.$ $\left.\mathrm{CH}_{2}, \mathrm{CH}_{2} \mathrm{CH}_{2} \mathrm{NH}\right), 1.53 \sim 1.44\left(\mathrm{~m}, 2 \mathrm{H}, \mathrm{SCH}_{2} \mathrm{CH}_{2} \mathrm{CH}_{2}\right)$, $1.00\left(\mathrm{t}, J=7.29 \mathrm{~Hz}, 3 \mathrm{H}, \mathrm{NHCH}_{2} \mathrm{CH}_{2} \mathrm{CH}_{3}\right), 0.94$ (t, $J=7.22$ $\left.\mathrm{Hz}, 3 \mathrm{H}, \mathrm{SCH}_{2} \mathrm{CH}_{2} \mathrm{CH}_{2} \mathrm{CH}_{3}\right) ;{ }^{13} \mathrm{C} \mathrm{NMR}\left(\mathrm{CDCl}_{3}, 100 \mathrm{MHz}\right)$ $\delta: 162.65,154.01,152.44,149.94,112.39,61.08,56.79$, $45.66,42.56,31.71,30.92,29.93,23.18,22.15,13.75$, 11.45; IR (KBr) v: 3449, 3299, 2925, 2862, 1618, 1463, 1415, 1324, 1143, 1060, 753, $727 \mathrm{~cm}^{-1}$; HRMS (ESI) calcd for $\mathrm{C}_{15} \mathrm{H}_{26} \mathrm{~N}_{5} \mathrm{O}_{2} \mathrm{~S}[\mathrm{M}+\mathrm{H}]^{+}$340.1807, found 340.1805 . 
9-(2-羟基乙基)-6-正丁氨基-8-甲氧基-2-正丁硫基 嘌呤 $\left(\mathbf{8 c}_{2}\right)$ : 白色固体, 产率 $92 \%$. m.p. $131 \sim 132{ }^{\circ} \mathrm{C} ;{ }^{1} \mathrm{H}$ NMR $\left(\mathrm{CDCl}_{3}, 400 \mathrm{MHz}\right) \delta: 5.35(\mathrm{~s}, 1 \mathrm{H}, \mathrm{NH}), 4.94(\mathrm{~s}, 1 \mathrm{H}$, $\mathrm{OH}), 4.09 \sim 4.07\left(\mathrm{~m}, 5 \mathrm{H}, \mathrm{CH}_{2} \mathrm{O}, \mathrm{OCH}_{3}\right), 3.96(\mathrm{t}, J=5.24$ $\mathrm{Hz}, 2 \mathrm{H}, \mathrm{NCH}_{2} \mathrm{CH}_{2} \mathrm{O}$ ), $3.63 \sim 3.59$ (q, $J=6.37 \mathrm{~Hz}, 2 \mathrm{H}$, $\left.\mathrm{CH}_{2} \mathrm{NH}\right), 3.10\left(\mathrm{t}, J=7.29 \mathrm{~Hz}, 2 \mathrm{H}, \mathrm{CH}_{2} \mathrm{~S}\right), 1.77 \sim 1.60(\mathrm{~m}$, $\left.4 \mathrm{H}, \quad \mathrm{SCH}_{2} \mathrm{CH}_{2}, \quad \mathrm{CH}_{2} \mathrm{CH}_{2} \mathrm{NH}\right), \quad 1.51 \sim 1.40(\mathrm{~m}, \quad 4 \mathrm{H}$, $\left.\mathrm{SCH}_{2} \mathrm{CH}_{2} \mathrm{CH}_{2}, \quad \mathrm{CH}_{2} \mathrm{CH}_{2} \mathrm{CH}_{2} \mathrm{NH}\right), 0.98 \sim 0.92(\mathrm{~m}, 6 \mathrm{H}$, $\left.\mathrm{NHCH}_{2} \mathrm{CH}_{2} \mathrm{CH}_{2} \mathrm{CH}_{3}, \quad \mathrm{SCH}_{2} \mathrm{CH}_{2} \mathrm{CH}_{2} \mathrm{CH}_{3}\right) ; \quad{ }^{13} \mathrm{C} \quad \mathrm{NMR}$ $\left(\mathrm{CDCl}_{3}, 100 \mathrm{MHz}\right) \delta: 162.64,154.02,152.54,149.96$, $112.35,61.33,56.84,46.88,45.89,31.66,30.91,22.14$, 20.09, 13.84, 13.75; IR (KBr) v: 3361, 3229, 2923, 2860, 1621, 1461, 1414, 1322, 1172, 1039, $728 \mathrm{~cm}^{-1}$; HRMS (ESI) calcd for $\mathrm{C}_{16} \mathrm{H}_{28} \mathrm{~N}_{5} \mathrm{O}_{2} \mathrm{~S}[\mathrm{M}+\mathrm{H}]^{+}$354.1964, found 354.1963.

9-(2-羟基乙基)-6-对氟苯乙氨基-8-甲氧基-2-正丁 硫基嘌呤 $\left(8 \mathbf{c c}_{3}\right)$ : 白色固体, 产率 $94 \%$. m.p. $145 \sim 146{ }^{\circ} \mathrm{C}$; ${ }^{1} \mathrm{H}$ NMR $\left(\mathrm{CDCl}_{3}, 400 \mathrm{MHz}\right) \delta: 7.11 \sim 6.99(\mathrm{~m}, 4 \mathrm{H}, \mathrm{Ph})$, 5.35 (s, 1H, NH), 5.13 (s, 1H, OH), 4.39 (t, $J=5.26 \mathrm{~Hz}$, $\left.2 \mathrm{H}, \mathrm{CH}_{2} \mathrm{O}\right), 4.29 \sim 4.27\left(\mathrm{~m}, 5 \mathrm{H}, \mathrm{NCH}_{2} \mathrm{CH}_{2} \mathrm{O}, \mathrm{OCH}_{3}\right.$ ), $3.71 \sim 3.66$ (q, $J=6.61 \mathrm{~Hz}, 2 \mathrm{H}, \mathrm{CH}_{2} \mathrm{NH}$ ), 3.18 (t, $J=7.29$ $\mathrm{Hz}, 2 \mathrm{H}, \mathrm{CH}_{2} \mathrm{~S}$ ), 2.91 (t, $J=6.88 \mathrm{~Hz}, 2 \mathrm{H}, \mathrm{CH}_{2} \mathrm{Ph}$ ), $1.78 \sim$ $1.70\left(\mathrm{~m}, 2 \mathrm{H}, \mathrm{SCH}_{2} \mathrm{CH}_{2}\right), 1.52 \sim 1.45\left(\mathrm{~m}, 2 \mathrm{H}, \mathrm{SCH}_{2} \mathrm{CH}_{2}-\right.$ $\mathrm{CH}_{2}$ ), 0.96 (t, $\left.J=7.39 \mathrm{~Hz}, 3 \mathrm{H}, \mathrm{SCH}_{2} \mathrm{CH}_{2} \mathrm{CH}_{2} \mathrm{CH}_{3}\right) ;{ }^{13} \mathrm{C}$ NMR $\left(\mathrm{CDCl}_{3}, 100 \mathrm{MHz}\right) \delta$ : 163.24, 160.15, 154.62, $152.18,150.53,135.12,130.27,130.19,124.10,115.41$, $115.20,61.66,56.82,46.52,42.48,39.98,35.51,31.91$, 22.18, 13.76; IR (KBr) v: 3482, 3346, 2934, 2865, 1620,
1467, 1416, 1330, 1151, 1053, 824, $727 \mathrm{~cm}^{-1}$; HRMS (ESI) calcd for $\mathrm{C}_{20} \mathrm{H}_{27} \mathrm{FN}_{5} \mathrm{O}_{2} \mathrm{~S}[\mathrm{M}+\mathrm{H}]^{+} 420.1869$, found 420.1868 .

\section{References}

[1] Jennings, L. K. Am. J. Cardiol. 2009, 103(3), 4.

[2] Camaioni, E.; Boyer, J. L; Mohanram, A. J. Med. Chem. 1998, $41(2), 183$

[3] Ingall, A. H.; Dixon, J.; Bailey, A. J. Med. Chem. 1999, 42(2), 213.

[4] Fox, S. C.; Behan, M. W. H.; Heptinstall, S. Cell Calcium 2004, $35(1), 39$.

[5] Owen, T. R; Serradell, N.; Bolos, J. Drugs Future 2007, 32(10), 845.

[6] Springthorpe, B.; Bailey, A.; Barton, P. Bioorg. Med. Chem. Lett. 2007, 17(21), 6013.

[7] Eltayeb, A.; Griessmeier, K. J.; Mueller, C. E. Bioorg. Med. Chem. Lett. 2005, 15(24), 5450.

[8] Mateos, T. G.; Evans, R. J.; Heath, M. F. Res. Vet. Sci. 2002, 73(2), 171.

[9] Vasiljev, K. S.; Uri, A.; Laitinen, J. T. Neuropharmacology 2003, $45(1), 145$.

[10] Zhang, Y.; Ye, J.; Hu, L. J. Thromb. Haemostasis 2012, 10(10), 2149.

[11] Kikugawa, K.; Iizuka, K.; Ichino, M. J. Med. Chem. 1973, 16(4), 358.

[12] Kikugawa, K.; Suehiro, H.; Ichino, M. J. Med. Chem. 1973, 16(12), 1381.

[13] Wu, Z. J.; Li, S. L.; Ding, Z. R.; Du, H. G. Chin. J. Org. Chem. 2011, 31(10), 1618 (in Chinese).

(吴兆军, 李顺来, 丁忠仁, 杜洪光, 有机化学, 2011, 31(10), 1618.)

[14] He, Q. W.; Du, H. G. Chin. J. Org. Chem. 2012, 32(9), 1678 (in Chinese).

(何琦文, 杜洪光, 有机化学, 2012, 32(9), 1678.) 